NOTA TÉCNICA

\title{
EVALUACIÓN AGRONÓMICA DE CEREALES FORRAJEROS DE USO POTENCIAL EN SISTEMAS DE PRODUCCIÓN DE LECHE DE ALTURA
}

\author{
María Mesén ${ }^{1}$, William Sánchez ${ }^{1}$
}

\begin{abstract}
RESUMEN
La presente investigación se realizó en el año 1996, en el distrito Cot, cantón Oreamuno, provincia Cartago. La topografía de la finca es irregular y se ubica a una altitud de $2.100 \mathrm{msnm}$. El objetivo del estudio fue evaluar la adaptabilidad de una colección de $13 \mathrm{ce}-$ reales forrajeros, nueve variedades de Avena (Avena sativa), tres de Trigo (un Triticum secale y dos Triticum aestivum) y una de Cebada (Hordeum vulgare). El diseño experimental utilizado, fue de bloques completos al azar, con tres repeticiones, representando cada cultivar un tratamiento. Las variables evaluadas fueron: cobertura, altura, plagas, producción de biomasa y valor nutritivo. En cuanto a las variables cobertura y altura los valores fueron altos en todos los cultivares cosechados, superiores a $90 \%$ y $75 \mathrm{~cm}$ respectivamente, los Triticum y la Hordeum vulgare fueron inferiores a las avenas en ambos aspectos. La única enfermedad que se presentó fue la Puccinia sp. eliminando por completo las avenas $\mathrm{Ne}$ huén, Dula, Gaviota y Llaofén y afectando levemente (5\% del follaje afectado) los cereales Culgoa II, Águila y Triticum secale. Los demás cereales no fueron dañados por ninguna plaga. La producción de biomasa en los diferentes cultivares osciló en un rango de 6,8 y 15,5 t/ha/corte. El análisis de varianza para esta variable mostró diferencias entre cultivares $\mathrm{P}=0,01$ y $\mathrm{P}=0,001$ para $\mathrm{t} / \mathrm{ha} / \mathrm{corte}$ y tasa de crecimiento respectivamente. Según los altos rendimientos de materia seca, adecuado valor nutritivo y ausencia de plagas, se puede concluir que los cereales que mejor se adaptaron a la zona en estudio fueron las avenas Riel, Nobby y Amby. Se debe continuar investigando con los cultivares sobresalientes bajo pastoreo y en sustitución de alimentos balanceados.
\end{abstract}

Palabras clave: cereales, forrajes, valor nutritivo, producción de leche.

\section{INTRODUCCIÓN}

La producción de leche en áreas tropicales depende en gran medida de los forrajes, principalmente gramíneas, las cuales siguen siendo la fuente alimenticia más común y de menor costo, sin embargo frecuentemente no proporcionan la cantidad y/o calidad que requieren los animales principalmente las vacas lecheras de mediana y alta producción.
En estos casos la mayoría de las empresas ganaderas llenan los requerimientos mediante el uso de alimentos balanceados, basados en materias primas importadas, lo cual encarece los costos de producción en el rubro de alimentación, representando éstos hasta un $72 \%$ de los costos totales de alimentación en ganadería de leche especializada (MAG; IICA 1991).

1 Instituto Nacional de Innovación y Transferencia en Tecnología Agropecuaria (INTA). Costa Rica. 
Por lo anteriormente mencionado, es de mucha importancia buscar variedades forrajeras con altos rendimientos y de buen valor nutritivo que puedan sustituir parcial o totalmente los alimentos balanceados.

El objetivo de este estudio fue evaluar una colección de cereales forrajeros para clima frío, con el fin de seleccionar variedades de gramíneas sobresalientes que se adapten a la zona alta lechera de Costa Rica.

\section{MATERIALES Y MÉTODOS}

El presente trabajo se desarrolló en el año 1996 en una finca ubicada en el distrito Cot, cantón Oreamuno, provincia Cartago, a $83^{\circ}$ 53' 30" longitud oeste y a 9o 57' latitud norte. La topografía de la finca es irregular y se ubica a una altitud de 2.100 msnm. La temperatura y precipitación promedio anual son de 21 ${ }^{\circ} \mathrm{C}$ y $1.857 \mathrm{~mm}$ respectivamente.

El terreno utilizado había sido cultivado anteriormente con forrajes de piso. Geomorfológicamente el suelo es de origen volcánico y taxonómicamente corresponde al orden de los andisoles, que son suelos de origen volcánico, característicos de la zona alta lechera de Costa Rica (Bertsch et al. 1993).

El análisis de suelo (Cuadro 1) presenta valores de $\mathrm{pH}$ y aluminio dentro del rango óptimo (según la guía para la interpretación de la fertilidad de suelo Bertsch (1987), como consecuencia el porcentaje de acidez es 8,8 . Borel (1981), menciona que los valores de acidez inferiores al $25 \%$ son adecuados para gramíneas. Los contenidos de $\mathrm{Ca}, \mathrm{Mg}, \mathrm{K}, \mathrm{P}$, los microelementos y las relaciones se encuentran en un rango óptimo, mientras que el Fe se encuentra en un nivel alto (Bertsch 1987).

La preparación del suelo fue mecánica, utilizando una arada, dos rastreadas y surcando cada $0,5 \mathrm{~m}$.

La siembra se realizó el 14 de setiembre de 1996 y la fase de campo terminó al inicio de la floración en las diferentes variedades, aproximadamente cinco meses después. Se utilizó semilla sexual de una colección de cereales forrajeros para clima frío (Cuadro 2).

Se hicieron evaluaciones de altura, cobertura, incidencia de plagas, producción de biomasa y valor nutritivo.

La metodología de evaluación que se utilizó fue una modificación de la utilizada por la Red Internacional de Evaluación de Pastos Tropicales (RIEPT) (Toledo1982).

\section{Descripción de la unidad experimental}

La parcela experimental fue de $6 \mathrm{~m}^{2}$ con tres hileras de $3,0 \mathrm{~m}$ de largo y distanciadas entre ellas a $0,5 \mathrm{~m}$. La unidad experimental fue la hilera central. El diseño experimental utilizado fue de bloques completos al azar con tres repeticiones, representando cada cultivar un tratamiento.

\section{Fertilización}

Las dosis aplicadas fueron $100,50,50$, 20 y $20 \mathrm{~kg} / \mathrm{ha}$ de nitrógeno, $\mathrm{P}_{2} \mathrm{O}_{5}, \mathrm{~K}_{2} \mathrm{O}, \mathrm{Mg}$ y $\mathrm{S}$. El fósforo, magnesio y azufre se incorporaron al suelo a la siembra, el potasio se aplicó

Cuadro 1. Resultado del análisis de suelo antes de la siembra. Cartago, 1996.1

\begin{tabular}{|c|c|c|c|c|c|c|c|c|c|c|c|}
\hline \multicolumn{5}{|c|}{ Meq/100ml suelo } & \multicolumn{5}{|c|}{ Ug/ml suelo } & \multirow[t]{2}{*}{ Textura } & \multirow{2}{*}{$\begin{array}{l}\text { Materia } \\
\text { orgánica }\end{array}$} \\
\hline pH & Al & $\mathrm{Ca}$ & Mg & $\mathbf{K}$ & $\mathbf{P}$ & $\mathrm{Zn}$ & Mn & $\mathrm{Cu}$ & $\mathrm{Fe}$ & & \\
\hline 6,0 & 0,15 & 7,8 & 2,0 & 1,5 & 15,0 & 19,9 & 13,0 & 32,0 & 208 & Franco & 11,3 \\
\hline
\end{tabular}

${ }^{1}$ Análisis de suelo realizado por el Laboratorio de Suelos, Foliares y Aguas del INTA. 
Cuadro 2. Cultivares de gramíneas que se evaluaron. Cartago, 1996.

\begin{tabular}{lcl}
\hline \multicolumn{1}{c}{ Cultivar } & Simbología & Procedencia \\
\hline Avena sativa, Nehuén & AN & Chile \\
Avena sativa, Amby & AA & Australia \\
Avena sativa, Culgoa II & AC & Australia \\
Avena sativa, Nobby & AB & Australia \\
Avena sativa, Dula & AD & Bolivia \\
Avena sativa, Gaviota & AG & Bolivia \\
Avena sativa, Águila & AU & Chile \\
Avena sativa, Llaofén & AL & Chile \\
Avena sativa, Riel & AR & Australia \\
Triticum secale, Lasko & TL & Inglaterra \\
Triticum aestivum, Axona & TA & Inglaterra \\
Triticum aestivum, Chablis & TC & Inglaterra \\
Hordeum vulgare, Hart & $\mathrm{CH}$ & Inglaterra \\
\hline
\end{tabular}

fraccionado, con 1/3 de lo recomendado cuatro semanas después de la siembra, 1/3 después del corte de uniformización y el último tercio al cabo de dos cortes (Toledo y Schultze-Kraft 1982).

\section{Variables a evaluar}

\section{Cobertura}

Esta variable se evaluó como porcentaje del área que no presenta suelo desnudo. Las evaluaciones se realizaron al inicio de la floración. Para este propósito, se utilizó la metodología propuesta por la RIEPT (Toledo y Schultze-Kraft 1982), con la diferencia de que al tener las parcelas solo tres hileras, de las cuales una constituye la parcela útil, se utilizó un marco rectangular de $0,5 \mathrm{~m}$ * $1,0 \mathrm{~m}$, con cuadrículas de $0,25 \mathrm{~m} * 0,25 \mathrm{~m}$.

\section{Altura}

Para esta medición, se tomó la altura desde el nivel del suelo hasta el punto más alto de la planta, sin estirarla y sin considerar la inflorescencia.

Plagas (Insectos y enfermedades) (Adaptado de Calderón 1982 y Lenné 1982)

Esta variable se evaluó cada dos semanas después de la siembra.
Se utilizó una escala de uno a cuatro en la cual:

$$
\begin{aligned}
& 1=\text { Presencia (5\% del follaje afectado) } \\
& 2=\text { Daño leve (5-20\% afectado) } \\
& 3=\text { Daño moderado (20-40\% afectado) } \\
& 4=\text { Daño grave (más de } 40 \% \text { afectado) } \\
& \text { Producción de biomasa y valor nutritivo }
\end{aligned}
$$

Las evaluaciones se realizaron al inicio de la floración, la altura de corte fue de $5 \mathrm{~cm}$ sobre el nivel del suelo. Los cortes se efectuaron a razón de un metro en la hilera central de cada parcela, dejando sin cortar los extremos, como efecto de borde (Roig 1989). El material de un metro de la hilera central se pesó en verde y luego una sub-muestra de $500 \mathrm{~g}$ se utilizó para la determinación del contenido de materia seca (MS), proteína cruda (PC) y digestibilidad in vitro de la materia seca (DIVMS).

\section{RESULTADOS Y DISCUSIÓN}

\section{Cobertura}

Todos los cultivares cosechados presentaron valores altos de cobertura, en un rango de 90 a $100 \%$ de suelo cubierto. Los Triticum y la Hordeum vulgare fueron inferiores a las avenas (Cuadro 3).

\section{Altura}

En cuanto a la altura, al inicio de la floración los valores oscilaron entre 85 y $111 \mathrm{~cm}$ y los Triticum y la Hordeum vulgare excepto el Triticum aestivum, Chablis presentaron también valores inferiores a los de las avenas (Cuadro 3).

\section{Plagas (Insectos y enfermedades)}

La única enfermedad que se presentó a partir de los 46 días de crecimiento y permaneció hasta el inicio de floración, fue la Puccinia $s p$, identificada por el Laboratorio de protección de cultivos del INTA. Dicha enfermedad eliminó por completo las avenas Nehuén, 
Dula, Gaviota y Llaofén y afectó levemente ( $5 \%$ del follaje afectado) a los cereales Culgoa II, Águila y Triticum secale. Los demás cereales no presentaron ninguna plaga.

\section{Producción de biomasa y valor nutritivo}

Los cereales se evaluaron al inicio de la floración, sin embargo presentaron diferencias en cuanto edad de maduración, por lo tanto fueron cosechados a diferentes edades; la Avena sativa, Culgoa II y la Hordeum vulgare, Hart a los 88 días de crecimiento, y los demás cultivares que sobrevivieron al ataque de Puccinia sp. se cortaron a los 130 días.

Con los resultados de producción de biomasa en base seca (Cuadro 3) se realizó el análisis de varianza, encontrándose diferencias entre cultivares $\mathrm{P}=0,01$ y $\mathrm{P}=0,001$ para $\mathrm{t} /$ ha/corte y tasa de crecimiento diaria respectivamente.

Los rendimientos de producción de biomasa en base seca (Cuadro 3) de las avenas fueron altos, presentaron valores en un rango de 12,3 y 15,5 t/ha/corte. De acuerdo con la prueba de Waller-Duncan (Figura 1) la Avena sativa, Riel fue la de mayor producción de materia seca 15,5 t/ha/corte; sin embargo no difiere significativamente de los otros cereales

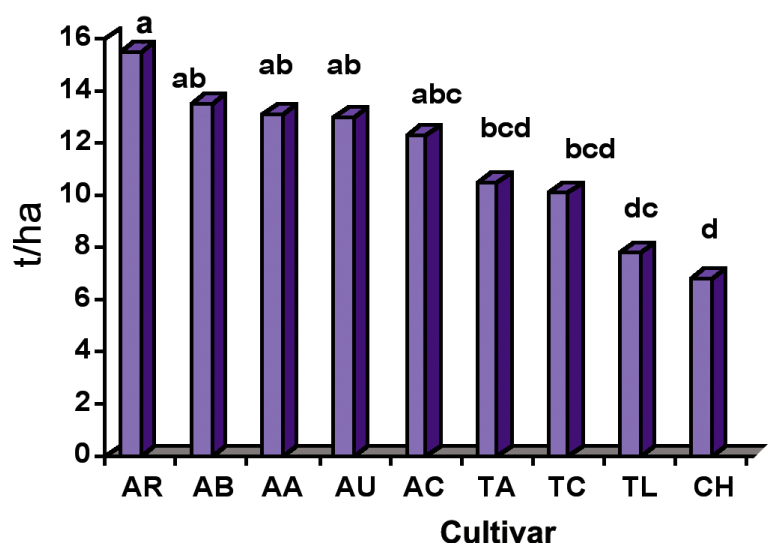

Figura 1. Producción de biomasa en base seca. Cartago, 1996.

Letras iguales no difieren entre sí $(P>0,05)$.

del mismo género. Sánchez (1998) encontró en la zona alta lechera de Costa Rica un valor promedio de 14,4 t/ha/corte en ocho cereales de Avena sativa, datos similares a los obtenidos en este estudio. Mesén (1997) reporta valores más altos $22,2 \mathrm{t} / \mathrm{ha}$ /corte en promedio para cinco cultivares de Avena sativa.

En cuanto a la tasa de crecimiento la Avena sativa, Culgoa II fue la que presentó un valor más alto $(139,8 \mathrm{~kg} / \mathrm{ha} /$ día $)$ y según la prueba de Waller Duncan (Figura 2) fue diferente significativamente a los demás cerea-

Cuadro 3. Comportamiento de los cereales al inicio de la floración. Cartago, 1996.

\begin{tabular}{|c|c|c|c|c|c|c|c|}
\hline Cultivar & $\begin{array}{c}\text { Días de } \\
\text { crecimiento }\end{array}$ & $\begin{array}{c}\text { Materia seca, } \\
\text { t/ha/corte }\end{array}$ & $\begin{array}{c}\text { Materia seca, } \\
\text { kg/ha/día }\end{array}$ & $\begin{array}{l}\text { Altura, } \\
\text { cm }\end{array}$ & $\begin{array}{c}\text { Cobertura, } \\
\%\end{array}$ & P.C., \% & DIVMS, \% \\
\hline $\mathrm{AN}^{*}$ & - & - & - & - & - & - & - \\
\hline AA & 130 & 13,1 & 100,8 & 97 & 100 & 13,3 & 59,9 \\
\hline$A C$ & 88 & 12,3 & 139,8 & 103 & 95 & 18,0 & 69,0 \\
\hline$A B$ & 130 & 13,5 & 103,8 & 102 & 100 & 15,9 & 70,0 \\
\hline$A D^{*}$ & - & - & - & - & - & - & - \\
\hline$A G^{*}$ & - & - & - & - & - & - & - \\
\hline $\mathrm{AU}$ & 130 & 13,0 & 100,0 & 104 & 100 & 13,0 & 61,9 \\
\hline$A L^{*}$ & - & - & - & - & - & - & - \\
\hline $\mathrm{AR}$ & 130 & 15,5 & 119,2 & 111 & 100 & 14,2 & 65,3 \\
\hline TL & 130 & 7,8 & 60,0 & 75 & 90 & 17,0 & 80,0 \\
\hline TA & 130 & 10,5 & 80,8 & 85 & 95 & 14,5 & 61,1 \\
\hline TC & 130 & 10,1 & 77,7 & 98 & 95 & 15,1 & 60,1 \\
\hline $\mathrm{CH}$ & 88 & 6,8 & 77,3 & 92 & 90 & 20,4 & 67,3 \\
\hline
\end{tabular}

*No aparecen datos porque son cultivares que se perdieron. 


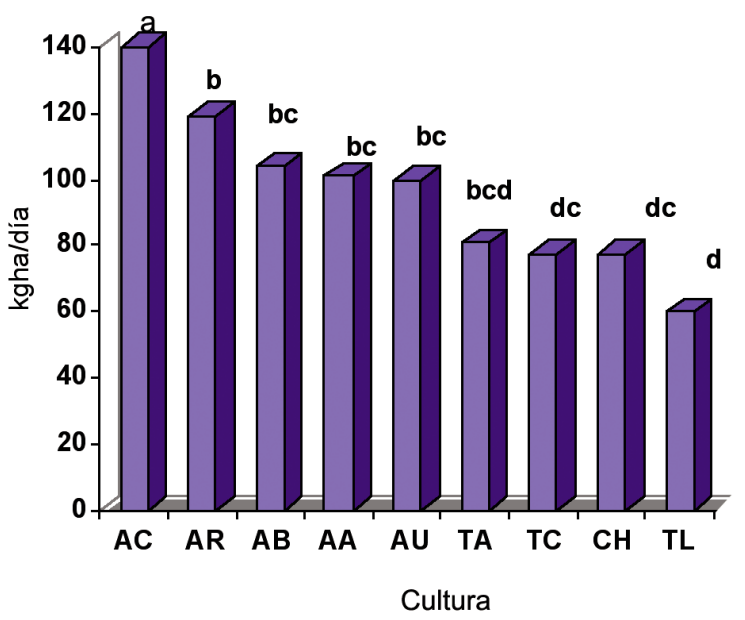

Figura 2. Tasa de crecimiento (kg/ha/día). Cartago, 1996.

Letras iguales no difieren entre sí $(P>0,05)$.

les; ésto se debe a que es un forraje de maduración temprana.

En cuanto al valor nutritivo (Cuadro 3) no se realizó análisis de varianza ya que por falta de presupuesto se analizaron muestras compuestas de las tres repeticiones.

Los datos reportados de valor nutritivo, demostraron que el cereal de mayor contenido de proteína cruda fue la cebada (Hordeum vulgare, Hart); sin embargo por poseer un rendimiento de materia seca inferior, presentó menor cantidad de proteína cruda por unidad de superficie que los demás cereales. Los forrajes con una mayor cantidad de proteína cruda y materia seca digestible por hectárea fueron los mismos cuya producción de biomasa fue mayor ya que los valores nutricionales fueron similares.

Sánchez (1998) menciona que el costo de la materia seca, proteína cruda y energía digestible proporcionada a los animales a través de la Avena sativa, es inferior a la suplida por los alimentos balanceados.

\section{CONCLUSIONES Y RECOMENDACIONES}

Teniendo en consideración las condiciones bajo las cuales se llevó a cabo el ensayo, se pueden formular las siguientes conclusiones y recomendaciones:

1. Los altos rendimientos en producción de biomasa de las avenas Riel, Nobby, Amby, Águila y Culgoa II demuestran la adaptabilidad de las mismas a la zona.

2. Los cereales Culgoa II, Águila y Triticum secale, Lasko fueron afectados levemente por Puccinia sp.

3. Las avenas Nehuén, Dula, Gaviota y Llaofén no se adaptan a la zona en estudio.

4. La Hordeum vulgare, Hart fue la variedad de menor producción por hectárea y de menor tasa de crecimiento.

5. Según los altos rendimientos de materia seca, adecuado valor nutritivo y ausencia de plagas, se puede concluir que los cereales que mejor se adaptan a la zona en estudio son las avenas Amby, Nobby y Riel.

6. Se debe continuar investigando con los cultivares sobresalientes bajo pastoreo y en sustitución de alimentos balanceados.

\section{AGRADECIMIENTO}

Los autores expresan su agradecimiento al Doctor Danilo Pezo Q., y a los Ingenieros Luis Villegas Z. y Beatriz Molina B. por la orientación científica que le brindaron a este trabajo. 


\section{LITERATURA CITADA}

BERTSCH, F. et. al. 1993. Características de los principales órdenes de suelos presentes en Costa Rica. Congreso Nacional Agropecuario y de Recursos Naturales San José. Costa Rica. Universidad de Costa Rica. 78 p.

BERTSCH, F. 1987. Manual para interpretar la fertilidad de los suelos de Costa Rica. San José, Costa Rica. Universidad de Costa Rica. $82 \mathrm{p}$.

BOREL, R. 1981. Uso de los fertilizantes en pasturas. In: Producción y utilización de forrajes en el trópico. Turrialba, Costa Rica. CATIE. p. 58-69

CALDERÓN, M. 1982. Evaluación del daño causado por insectos. In: Toledo, J.M. ed. Manual para la evaluación agronómica. Red Internacional de Evaluación de Pastos Tropicales (RIEPT). CIAT. Cali, Colombia. p. 45-56.

LENNE , J. 1982. Evaluación de enfermedades en pastos tropicales. In:Toledo, J.M. Manual para la evaluación agronómica. Red Internacional de Evaluación de Pastos Tropicales. (RIEPT). CIAT. Cali, Colombia. p. 57-72.

MAG (Ministerio de Agricultura y Ganadería); IICA (Instituto Interamericano de Cooperación para la Agricultura, CR). 1991. Estudio a nivel nacional para mejorar la tecnología de alimentación de ganado lechero. San José, Costa Rica. p. 265.

MESÉN, M. 1997. Evaluación del rendimiento y valor nutritivo de cinco variedades de avena forrajera (Avena sativa). Investigación Agrícola. p. 5-11.

MESÉN, M. 1997. Efecto de diferentes densidades de siembra y niveles de nitrógeno en avena Llaofén (Avena sativa). Investigación Agrícola 6: 33 -37.

ROIG, C.A. 1989. Evaluación preliminar de 200 accesiones de leguminosas forrajeras tropicales en el ecosistema de Bosque Tropical Lluvioso en Costa Rica. Guápiles, Costa Rica. Tesis Mag. Sc. Turrialba ,Costa Rica. CATIE. 179 p.

SÁNCHEZ, W. 1998. Evaluación de 13 cereales forrajeros de corte. Tesis Mag. Sc. San José. Costa Rica. UNED. 80 p.

TOLEDO, J.M.; SCHULTZE - KRAFT, R. 1982. Metodología para la evaluación agronómica de pastos tropicales. In: Toledo, J.M. Manual para la Evaluación Agronómica. Red Internacional de Evaluación de Pastos Tropicales (RIEPT). CIAT. Cali, Colombia. p. 91-109.

TOLEDO, J.M. 1982. Manual para la Evaluación Agronómica. Red Internacional de Evaluación de Pastos Tropicales (RIEPT). CIAT. Cali, Colombia. 166 p. 